\title{
NEURILEMOMA RETROPERITONEAL
}

\author{
E. ARGÜELlES SALIDO, C.B. CONGREGADO RUIZ, R.A. MEDINA LÓPEZ, \\ J.L. PASCUAL DEL POBIL MORENO
}

Servicio de Urología. Unidad de Uro-Oncología. Hospital Universitario Virgen del Rocío. Sevilla.

Actas Urol Esp. 28 (8): 628-630, 2004

\section{RESUMEN}

NEURILEMOMA RETROPERITONEAL

$\mathrm{El}$ schwanoma es un tumor poco frecuente, que tiene su origen en las envueltas neurales de los nervios periféricos. Para su diagnóstico es fundamental el hallazgo histológico de zonas Antoni A y B.

Presentamos un nuevo caso de localización retroperitoneal, que no es su situación más frecuente, en un varón de 60 años, que presentó dolor en flanco izquierdo. Recibió tratamiento quirúrgico, siguiendo una evolución favorable.

PALABRAS CLAVE: Tumor retroperitoneal. Neurilemoma. Schwannoma.

\section{ABSTRACT \\ RETROPERITONEAL NEURILEMMONA}

Schwannoma is an uncommon tumor arising from neural sheath of peripheral nerves. Histological findings showing Antoni A and B zones are the main diagnostic features.

We report a new case of a retroperitoneal located one, which is not its most usual place to appear, in a 60 years old male, presenting left flank pain. He recieved surgical treatment, having a satisfactory evolution.

KEY WORDS: Retroperitoneal tumor. Schwannoma. Neurilemmoma.

$\mathrm{L}$ os tumores primitivos del retroperitoneo no son neoformaciones frecuentes, alcanzando una incidencia máxima que oscila entre 0,2-0,6\% de todas las neoplasias del organismo. La presencia de tejido adiposo, estructuras vasculares, linfáticas, musculares del sistema nervioso periférico y vegetativo etc., van a constituirse en puntos de partida de diversas neoformaciones que se han intentado clasificar desde muy distintos puntos de vista y criterios: benignidad-malignidad, morfología y, el más aceptado, según su tejido de origen.

Presentamos un nuevo caso de neurilemoma retroperitoneal pararrenal diagnosticado de manera incidental. Las referencias en la literatura española no son frecuentes ya que el caso aportado viene a ser el décimo en la literatura que hemos alcanzado a consultar ${ }^{1-10}$.

\section{CASO CLÍNICO}

Varón de 60 años en estudio por el Servicio de Reumatología por poliartrosis y dolor en flanco izquierdo. Otros antecedentes personales destacables eran diabetes mellitus no insulinodependiente, fumador de un paquete/día. Analíticamente sólo destacaba una glucemia de $199 \mathrm{mgr} /$ $\mathrm{dL}$, con hemoglobina glucosilada de $8,5 \%$.

En radiografía simple se observan calcificaciones irregulares y aumento de tamaño del polo superior del riñón izquierdo, motivo por el que se 
realizó posteriormente una tomografía axial computerizada (TAC), evidenciándose masa hipodensa en dicha zona renal, con múltiples calcificaciones en su interior, que se diferenciaba bien de la glándula suprarrenal, siendo informado como posible hipernefroma renal.

Tras un estudio de extensión con resultados negativos se realiza exéresis quirúrgica de masa retroperitoneal mediante lumbotomía, que se evidenció independiente del riñón izquierdo.

El postoperatorio cursó sin incidencias, dándosele el alta al cuarto día del mismo.

Anatomopatológicamente fue informado como tumor nodular de $11 \times 7,5 \mathrm{~cm}$., con extensas áreas de calcificación con metaplasia ósea, y otras degenerativas. Mostraba células fusiformes con núcleos ovalados dispuestas en haces (áreas de Antoni A), con algunas atipias. Presentaba inmunorreactividad para vimentina y proteína S100. (Figs. 1 y 2). Con todo ello el diagnóstico era neurilemoma (Schwanoma) degenerado ("viejo").

Dos años después continúa asintomático, sin que se haya evidenciado recidiva tumoral.

\section{DISCUSIÓN}

El neurilemoma o schwanoma benigno es un tumor de origen neural que tiene su inicio en las células de Schwann que conforman la vaina nerviosa, pero sin relacionarse con el eje central del nervio ${ }^{1}$. En 1910 Verocay describe el primer tumor originado en un nervio periférico ${ }^{11}$. El término Schwanoma fue establecido por Masson $(1932)^{12}$, siendo Stout (1935) quien introdujo el término de neurilemoma ${ }^{13}$. Otras denominaciones que ha recibido han sido neurinoma, glioma periférico o fibroblastoma perineural.

Estos tumores suelen tener su procedencia en los distintos nervios periféricos de cabeza, cuello y extremidades, y con menos frecuencia mediastino o retroperitoneo. No obstante se han descrito schwanomas de localización renal, testicular, de cordón espermático, de túnica vaginal $^{2,3}$.

Es un tumor que puede desarrollarse a cualquier edad y sin preferencia por alguno de los sexos, aunque sí suelen tener mayor incidencia entre los 30-60 años. Actualmente su frecuencia entre los tumores retroperitoneales se estima entre el 1-10\% $\%^{3,14,15}$.
Ha sido descrita su asociación a la enfermedad de Von Recklinhausen, junto a los neurofibromas, en cuyo caso suelen ser malignos y de localización atípica ${ }^{1,10,14}$.

Clínicamente su sintomatología suele derivar de la compresión de estructuras y órganos vecinos ${ }^{4,15}$, aunque existe la posibilidad muy excepcional de presentación en forma de síndrome paraneoplásico consistente en una hipercalcemia en relación con una elevación de prostaglandinas, en especial la PGE2 ${ }^{16}$.

En su forma benigna, que suele ser darse el $70 \%$ de las ocasiones ${ }^{4}$, suele mostrarse, la mayoría de las veces, encapsulado, con aspectos sólido, quístico o mixto. Por el contrario, en su forma maligna, lo hace de manera difusa, con tendencia a invadir estructuras vecinas, lo que hace más complejo el acto quirúrgico. Es en el retroperitoneo donde suele alcanzar sus mayores dimensiones, y cuando se expresan clínicamente lo hacen con síntomas derivados de una masa ocupante de espacio.

Desde un punto de vista histológico los neurilemomas muestran un patrón de áreas estructurales distintas que se identifican como de Antoni A y Antoni B. La primera expresa una hipercelularidad sólida y núcleos en empalizada que son conocidos como cuerpos de Verocay. Las áreas B son hipocelulares, adoptando una textura histológica laxa ${ }^{1,2}$. Inmunohistoquímicamente se caracterizan por la positividad de la proteína S100, así como a la vimentina, tinción específica de su origen neural. Los neurofibromas, por el contrario, carecen de esta duplicidad estructural o de áreas histológicas ya que están formados por un patrón celular uniforme.

La punción aspiración con aguja fina (PAAF), cuando ha sido utilizada, ha mostrado, una vez más, su insuficiencia para la identificación diagnóstica. Las técnicas de imagen actuales excepcionalmente podrán conducir a un diagnóstico de sospecha histológica preoperatoria, aunque sí su topografía retroperitoneal. La apariencia de los neurilemomas en la TAC ha sido descrita como masas bien delimitadas homogéneas en scanner sin realce, y heterogénea con fuerte realce en anillo tras la administración de contraste. Estas áreas de realce se deben a zonas de cambios quísticos y hemorrágicos. La dife- 


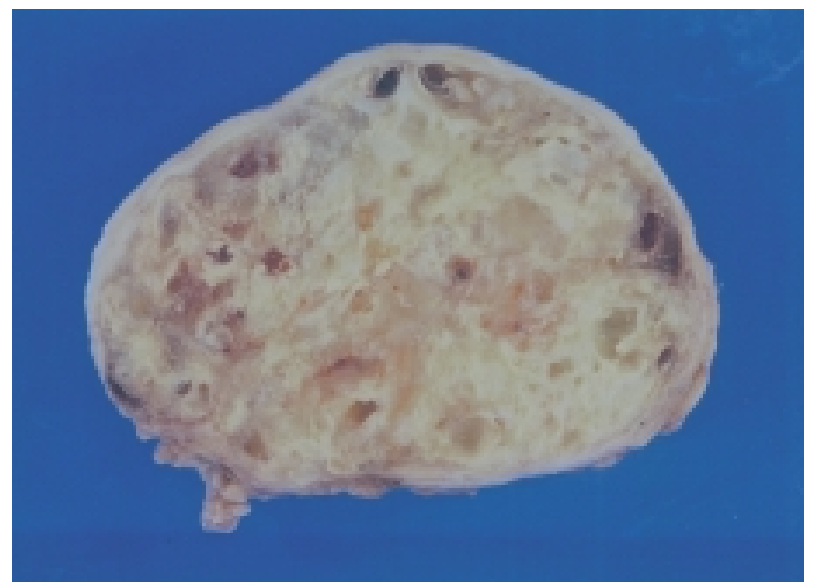

FIGURA 1. Tumor nodular con áreas de calcificación con metaplasia ósea y zonas degenerativas.

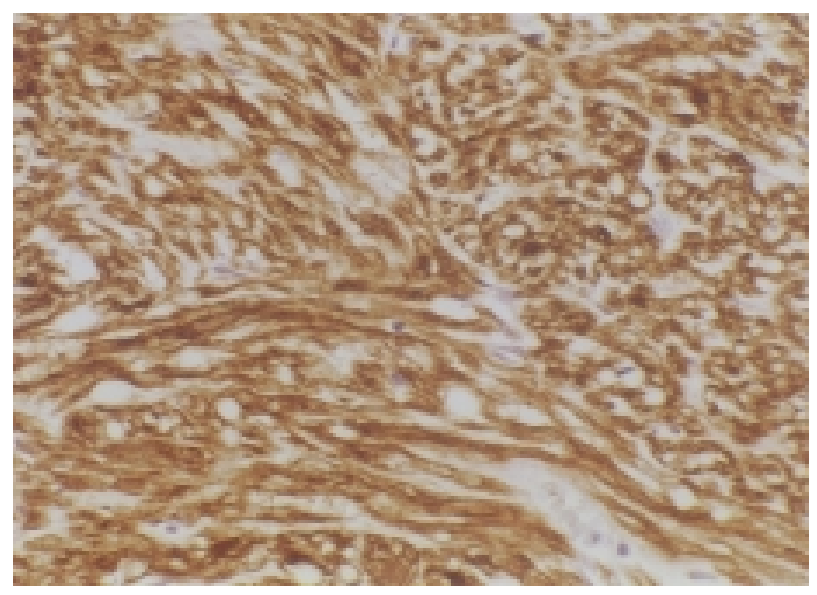

FIGURA 2. Inmunohistoquimica: positividad para S-100 en áreas fusiformes. Vimentina positiva. Cambios hialinos.

rencia de apariencia según se administre contraste o no ayuda al diagnóstico diferencial con el neurofibroma, en el que no se aprecian estas variaciones al no presentar el fenómeno de degeneración quística o hemorrágica. En la resonancia magnética nuclear (RMN) las masas presentan baja intensidad de señal en $\mathrm{T} 1 \mathrm{y}$ alta intensidad de señal en ${ }^{1}{ }^{17}$.

Lógicamente no existe otro tratamiento que el quirúrgico. A diferencia del neurofibroma su enucleación quirúrgica suele ser sencilla porque las fibras nerviosas no cruzan la lesión, sino que discurren por su cápsula ${ }^{1,17}$. No obstante, y a pesar de su carácter de benignidad histológica, ello no nos debe hacer olvidar la necesidad de revisiones periódicas al menos durante los primeros años.

\section{REFERENCIAS}

1. Sanroma I, Aurteneche JJ, Acinas O, Gurtubay I. Schwanoma retroperitoneal benigno. Arch Esp Urol 1993; 46: 513.

2. De Diego E, Roca A, Martín B et al. Schwanoma benigno retroperitoneal: aportación de un nuevo caso. Actas Urol Esp 2000;24(8):685-688.

3. Barrero R, Ramírez A, Morales A, Maestro JL, Huesa I, León E, Leal E, Moyano JL, García JL. Schwannoma benigno suprarrenal. Arch Esp Urol 2002;55:858-860.

4. Cortes I, Fernández M, De Castro F, Díaz J, Delgado M. Schwannoma retroperitoneal benigno: revisión de la literatura y aportación de un nuevo caso. Actas Urol Esp 1999;23:621-624.

5. Jiménez Cruz JF, Conejero J, Solé FJ. Neurinoma retroperitoneal. Arch Esp Urol 1977;30:267.

6. Rodríguez J, Sanfeliú F, Pujol F, Chavarría J, Serrallach N. Tumor retroperitoneal infrecuente: schwanoma. A propósito de un caso. Actas Urol Esp 1982;6:99-102.

7. Navalón P, Picurelli L, Ferrer Jiménez R, Farin M, Bataller J, Ferrer Roda JJ. Schwanoma retroperitoneal: aportación de un caso. Arch Esp Urol 1989;42:366-339.

8. Beardo P, Gutiérrez del Pozo R, Mallofre C et al. Schwanoma retroperitoneal benigno bilobulado. Arch Esp Urol 2001; 34:170.

9. Esclarín MA, Sanz JI, Ortas JL, Mengual P, Vera J, Abad J, Carmen D. Tumor retroperitoneal infrecuente: schwannoma benigno. Actas Urol Esp 1991;15:577-579.

10. Caraffaratti J, Hocsman H, Algaba F. Schwanoma benigno retroperitoneal. Act Fund Puigvert 1991;10:3.

11. Verocay J. Zur kenntnis der Neurofibrome. Beitr Pathol Anat Allg Pathol 1910;48:1-5.

12. Masson P. Experimental and spontaneous schwanomas. Am J Pathol 1932;8:367-370.

13. Stout AP. The peripheral manifestations of the specific nerve sheath tumor (neurilemmoma). Am J Cancer 1935, 24:51-55.

14. Hurley L, Smith JL, Larsen CR, Silverman ML. Multiple retroperitoneal schwannomas: case report and review of the literature. J Urol 1994;151:413-416.

15. Micaeli S, Virgili G, Vespasiani G, Silecchia A, D'Alessandro P, Micaeli F. Benign schwannoma surrounding and obstructing the ureteropelvic junction. Eur Urol 1997;32: 121-123.

16. Komiya I, Yamaguchi K, Yoshio M, Honda S, Tsuchihashi T, Shimizu T, Fukushima N, Saito Y. Retroperitoneal neurilemoma presenting with hypercalcemia associates with markedly elevated plasma prostaglandin levels. Cancer 1991;68:1086-1091.

17. Kim SH, Choi BI, Han Mc, Kim YI. Retroperitoneal neurilemoma: CT and MR findings. Am J Roentgenol 1992;159: 1023-1026.

\section{Dr. E. Argüelles Salido}

Servicio de Urología. Unidad de Uro-Oncología

Hospital Univ. Virgen del Rocío

Avda. Manuel Siurot, s/n - 41013 Sevilla

earguelles@telefonica.net

(Trabajo recibido el 2 septiembre de 2003) 